\title{
Generalized Spectral Analysis of Planar Lines on Layered Media Including Uniaxial and Biaxial Dielectric Substrates
}

\author{
FRANCISCO MEDINA, MANUEL HORNO, MEMBER, IEEE, AND HENRI BAUDRAND, MEMBER, IEEE
}

\begin{abstract}
In this paper the spectral-domain analysis (SDA) is generalized in order to compute the dispersive properties of a wide variety of planar and quasi-planar transmission lines (microstrips and finlines) printed on a stratified dielectric medium. Uniaxial and biaxial dielectric anisotropy can be easily manipulated due to the definition of a "transverse propagation matrix" characterizing each dielectric layer. The whole boundary value problem is reduced to two simpler problems involving only one or two dielectrics. Then, the spectral dyadic Green's function is derived via a recurrence algorithm. The dispersion equation is derived by using the Ritz-Galerkin method. The numerical convergence is substantially improved taking into account the asymptotic behavior of the series. A number of illustrative examples have been included to emphasize the power of the method.
\end{abstract}

\section{INTRODUCTION}

A CCURATE knowledge of the propagation parameters of printed lines plays a vital role in the design of modern microwave and millimeter-wave integrated circuits. Microstrip and finline configurations, the most widely used transmission lines for this range of frequencies, are becoming more and more complex, so it is desirable to include in any analysis the presence of several dielectric layers and/or coupled strips or fins. On the other hand, since certain materials used as substrates in those circuits exhibit dielectric anisotropy (occurring naturally or being introduced during the manufacturing process), it is therefore interesting to include this contingency. The importance of accounting for the anisotropy has been emphasized in a comprehensive review paper by Alexopoulos [1]. In this paper and the references therein, the reader can find most of the major contributions on planar lines with anisotropic substrates published prior to 1985.

The multiple boundary value problem appearing when we have a number of planar conductors embedded in a stratified dielectric medium has been examined under the quasi-TEM assumption using different analytical techniques (a detailed review of the literature on this subject is

Manuscript received April 20, 1988; revised September 13, 1988. This work was supported by the C.I.C.Y.T. (Project PB86-0144), Spain.

F. Medina and M. Horno are with the Departamento de Electricidad y Electrónica, Facultad de Física, Universidad de Sevilla, 41012 Sevilla, Spain.

H. Baudrand is with the Laboratoire de Microondes, Institute $\mathrm{Na}$ tionale Polytechnique de Toulouse, Toulouse, France.

IEEE Log Number 8825384 included in [2]). Cases involving anisotropic dielectrics have also been treated, for instance, in [3]. Unfortunately, the validity of these analyses is restricted to microstrip-like structures operating at the lower end of the microwave spectrum. The dispersive properties of these lines cannot be inferred from this type of model, and the analysis cannot be applied to non-TEM configurations such as finlines. Therefore, a full-wave analysis is required to adequately model their behavior. A number of techniques capable of dealing with planar structures with isotropic and/or anisotropic dielectrics using a dynamic model have already been described. These include the finite difference technique [4], the least square boundary residual method [5], the singular integral equation method [6], the method of lines [7], the generalized transverse resonance method [8], mode matching [9], the transmission line matrix method [10], and the Wiener-Hopf technique [11]. Nevertheless, the spectral-domain technique (SDA) is the most widely used, because of its feasibility in manipulating multilayer structures with one or several conducting strips [12], [13] or fins [14], [15].

In order to apply the SDA, the spectral dyadic Green's function must be calculated for the structure to be analyzed. To our knowledge, three different systematic methods to do this have been given in the literature, i.e., the spectral-domain immittance approach [16], the transfer matrix approach [17], and, more recently, an iterative algorithm based on the use of vector potentials and equivalent transmission line problems [18]. All these methods consider isotropic materials, although the concept of equivalent transverse transmission lines is extended in [19] to uniaxial anisotropic dielectrics and recently has been applied to the analysis of a unilateral finline with uniaxial substrate [20] in the SDA context. Maia et al. apply the SDA to microstrips and finlines on uniaxial substrates [21] using an extension of the Hertz potential treatment proposed by Lee and Tripathi [22]. A very general approach for complex anisotropic layered media using a four-component formulation (which yields first-order partial differential equations) is discussed from a theoretical point of view by Krowne [23]. The dispersive character of the fundamental modes of open microstrip and slotlike structures on anisotropic substrates is treated by introducing

0018-9480/89/0300-0504\$01.00 C1989 IEEE 
some transformed fields and using second-order differential equations [24], [25]. A fourth-order formulation is presented in [26] to analyze a bilateral finline on a biaxial anisotropic dielectric substrate. The extension of this method to a general multilayer problem is not straightforward.

The approach presented in this paper tries to overcome the difficulties inherent in the analysis of multilayer structures involving uniaxial or biaxial anisotropic dielectrics, and also makes use of the SDA. The spectral dyadic Green's function is obtained via a recurrence algorithm similar to the one reported in [27] for the quasi-static case. The algorithm is based on the definition of a "transverse propagation matrix" associated with each dielectric layer and the reduction of the whole problem to simpler singleor two-layer partial problems. The method leads to second-order differential equations, in contrast to [23] or [26]. The dispersion equation is achieved via the standard Ritz-Galerkin method. The asymptotic behavior of the dyadic Green's function and the Fourier transforms of the basis functions (used to approximate the surface charge density on the strips or the tangential electric fields in the slots) is explicitly incorporated in the computer programs, resulting in a drastic improvement of the convergence of the series defining the entries of the Ritz-Galerkin matrix. The theory developed in the paper has been used to write a computer program to find the dispersion characteristics (dominant and first higher order modes) of multistrip or multifin configurations embedded in a layered dielectric medium including uniaxial and biaxial anisotropic dielectrics. These programs have been conveniently checked with previously published results.

\section{Statement of the Problem}

Many planar transission lines used in practice can be viewed as particular cases of the general geometry shown in Fig. 1. This structure is assumed to be uniform in the $z$ direction and it consists of a number of printed conductors embedded in a layered lossless dielectric medium, the whole being enclosed by rectangular boundaries. The metallization thickness is negligible, and the electrical properties of the nonmagnetic dielectric materials will be described by the following diagonal tensor:

$$
\epsilon_{0} \overline{\bar{\epsilon}}=\epsilon_{0}\left(\epsilon_{x} \hat{x} \hat{x}+\epsilon_{y} \hat{y} \hat{y}+\epsilon_{z} \hat{z} \hat{z}\right) .
$$

It is possible to take into account both isotropic and uniaxial or biaxial anisotropic materials with optical axes normal to region interfaces (materials are often supplied or selected with this orientation). This, together with the treatment of the multilayer problem using a "transverse propagation matrix," is the main feature of the analysis carried out in this paper.

The rectangular boundary conditions assumed in Fig. 1 correspond to actual metallic enclosures or to electric/ magnetic walls induced by the symmetries of the structure and the propagation mode. It is clear that single and
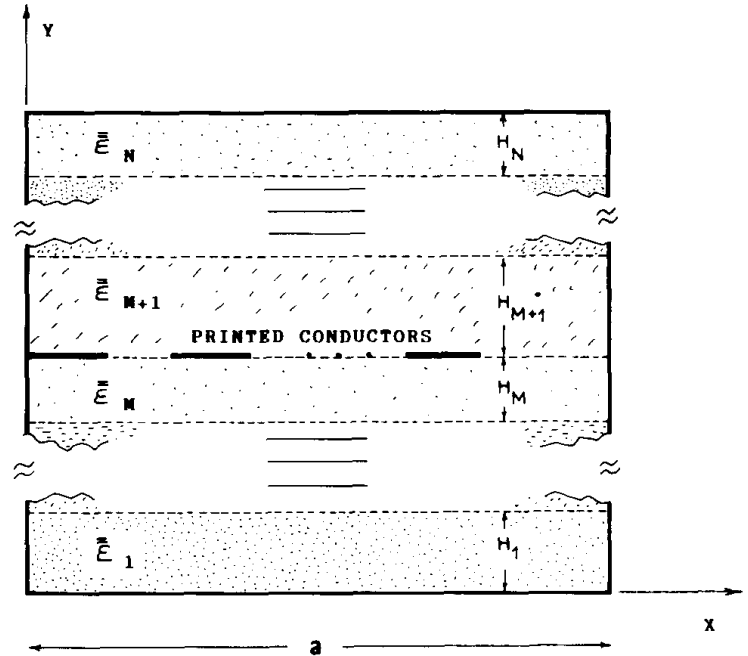

Fig. 1. Cross section of the generalized planar transmission line studied in this paper. The system is composed of $N$ isotropic or uniaxial/biaxial dielectric layers with conductors printed on the $M$ th interface. The rectangular enclosure represents electric or magnetic walls.

coupled microstrip lines, slotlines, and finlines can be modeled by the proposed general configuration. Our purpose is to provide a unified method to find the dispersive behavior of the propagation constant for this kind of transmission system in such a manner that the dielectric anisotropy and the number of dielectric layers or coupled lines are no longer a problem.

\section{Spectral ANalysis}

A full-wave analysis of the frequency-dependent behavior of the planar transmission system shown in Fig. 1 requires solving the Helmholtz equation subject to the appropriate boundary conditions. For each dielectric layer (see Fig. 2(a)) the electric field vector $(\vec{E})$ must obey the following equations:

$$
\begin{aligned}
\vec{\nabla}(\vec{\nabla} \cdot \vec{E})-\nabla^{2} \vec{E} & =k_{0}^{2 \overline{\bar{\varepsilon}}} \vec{E} \\
\vec{\nabla} \cdot(\overline{\bar{\epsilon}} \vec{E}) & =0
\end{aligned}
$$

with

$$
k_{0}^{2}=\omega^{2} \mu_{0} \epsilon_{0} .
$$

As stated above, the SDA has proved to be especially suitable in treating the multiple boundary problems we are interested in. Assuming time harmonic operation, each of the field quantities can be expressed as follows:

$$
A(x, y, z)=\sum_{n=-\infty}^{+\infty}\left\{\tilde{A}(n, y) e^{-j \alpha_{n} x}\right\} e^{-j \beta z}
$$

where $\tilde{A}(n, y)$ is the Fourier transform of $A$ in the $x$ direction, $\alpha_{n}$ is a Fourier variable, and $\beta$ is the unknown propagation constant.

Equations (2) can be rewritten in the spectral domain as a set of ordinary differential equations for the Fourier 


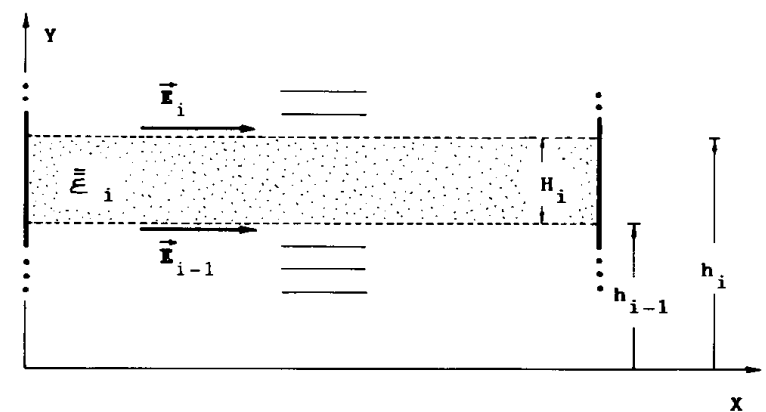

(a)

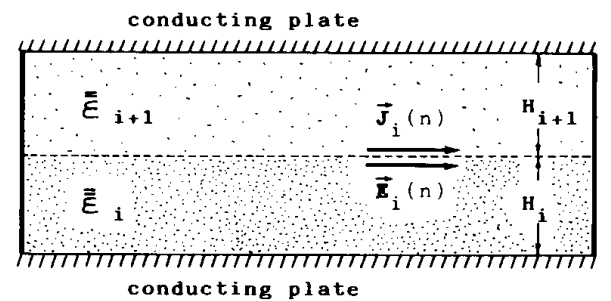

(b)

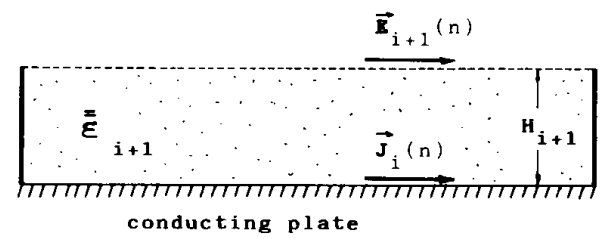

(c)

Fig. 2. (a) $i$ th dielectric layer of the structure in Fig. 1. (b) Two-layer structure to calculate $\overline{\bar{g}}_{i, i}(n)$. (c) Single-layer to calculate $\overline{\bar{g}}_{i, i+j}(n)$.

transforms of the electric field components:

$$
\begin{array}{rlr}
\frac{d^{2}}{d y^{2}} \tilde{\vec{E}}_{i} & =\overline{\bar{B}}_{i} \cdot \tilde{\vec{E}}_{i} \\
\overline{\bar{B}}_{i} & =\left[\begin{array}{cc}
\gamma_{x_{i}}^{2} & \alpha_{n} \beta \epsilon_{z y}^{* i} \\
\alpha_{n} \beta \epsilon_{x y}^{* i} & \gamma_{z i}^{2}
\end{array}\right] \quad \tilde{\vec{E}}_{i}=\left[\begin{array}{c}
\tilde{E}_{x i} \\
\tilde{E}_{z i}
\end{array}\right] \\
\gamma_{y i}^{2} \tilde{E}_{y i} & =j \frac{d}{d y}\left\{\alpha_{n} \tilde{E}_{x i}+\beta \tilde{E}_{z i}\right\}
\end{array}
$$

where

$$
\begin{aligned}
& \gamma_{x i}^{2}=\beta^{2}+\epsilon_{x y}^{i} \alpha_{n}^{2}-\epsilon_{x}^{i} k_{0}^{2} \\
& \gamma_{y i}^{2}=\beta^{2}+\alpha_{n}^{2}-\epsilon_{y}^{i} k_{0}^{2} \\
& \gamma_{z i}^{2}=\epsilon_{z y}^{i} \beta^{2}+\alpha_{n}^{2}-\epsilon_{z}^{i} k_{0}^{2}
\end{aligned}
$$

and

$$
\begin{array}{ll}
\epsilon_{x y}^{i}=\epsilon_{x} / \epsilon_{y} & \epsilon_{x y}^{* i}=\epsilon_{x y}^{i}-1 \\
\epsilon_{z y}^{i}=\epsilon_{z} / \epsilon_{y} & \epsilon_{z y}^{* i}=\epsilon_{z y}^{i}-1 .
\end{array}
$$

Note that the electric anisotropy is responsible for the coupling in (4a). Isotropic materials yield decoupled equations.
Let us center our attention on the $i$ th dielectric layer of the structure (Fig. 2(a)). The general solution of (4a) in the $i$ th dielectric layer can be expressed in terms of the spectral two-component electric vectors parallel to the region interfaces delimiting this region: $\left(\tilde{\overrightarrow{\vec{E}_{i}}}, \tilde{\vec{E}}_{i-1}\right)$. Using matrix notation,

$$
\begin{aligned}
\tilde{\vec{E}}_{i}(n, y)=\frac{\sinh \left[\overline{\bar{K}}_{i}\left(y-h_{i-1}\right)\right]}{\sinh \left[\overline{\bar{K}}_{i} H_{i}\right]} \\
\cdot \tilde{\vec{E}}_{i}-\frac{\sinh \left[\overline{\bar{K}}_{i}\left(y-h_{i}\right)\right]}{\sinh \left[\overline{\bar{K}}_{i} H_{i}\right]} \cdot \tilde{\vec{E}}_{i-1}
\end{aligned}
$$

with

$$
\tilde{\vec{E}}_{i}(n, y)=\left[\begin{array}{c}
\tilde{E}_{x i} \\
\tilde{E}_{z i}
\end{array}\right] \quad \dot{\overrightarrow{E_{i}}}=\tilde{\vec{E}}_{i}\left(n, y=h_{i}\right)
$$

where we have defined a transverse propagation matrix related to each dielectric layer:

$$
\overline{\bar{K}}_{i}=\left(\overline{\bar{B}}_{i}\right)^{1 / 2} \text {. }
$$

This matrix reduces to the scalar transverse propagation constant given by Itoh [14] for isotropic materials.

Note that the entire problem can be subdivided into a number of simpler two-layer problems corresponding to each pair of adjacent dielectrics. A surface current distribution, $\tilde{\overrightarrow{\vec{J}}}(n)$, lying at the $i$ th interface is linearly related to the horizontal components of the electric fields at the $(i-1)$ th, $i$ th, and $(i+1)$ th interfaces. In the spectral domain this relation can be written in a very simple manner:

$j \omega \mu_{0} \tilde{\overrightarrow{\vec{J}}}(n)=\overline{\bar{g}}_{i, i-j}(n) \cdot \tilde{\vec{E}}_{i-j}+\overline{\bar{g}}_{i, i}(n) \cdot \tilde{\vec{E}}_{i}+\overline{\bar{g}}_{i, i+1}(n) \cdot \tilde{\vec{E}}_{i+1}$

where

$$
\tilde{\overrightarrow{J_{i}}}(n)=\left[\begin{array}{c}
\tilde{J_{x}} \\
\tilde{J_{z}}
\end{array}\right] .
$$

(In the space domain these expressions involve complex convolution integrals.)

Our problem reduces now to one of determining the $\overline{\bar{g}}$ matrices. Fortunately, these can be easily derived from the solution of two simple problems involving only one or two dielectric layers.

\section{A. Evaluation of $\overline{\bar{g}}_{i, i}$}

In order to find $\overline{\bar{g}}_{i, i}$ we must solve the problem shown in Fig. 2(b). The tangential electric field at the interface between the $i$ th and $(i+1)$ th dielectric layers is related to the surface current at this interface as follows:

$$
j \omega \mu_{0} \tilde{\overrightarrow{\vec{J}}}_{i}=\overline{\bar{g}}_{i, i}(n) \cdot \tilde{\vec{E}}_{i} .
$$


The tangential magnetic field in the spectral domain is derived from the electric field for each layer via

$$
j \omega \mu_{0} \tilde{\vec{H}}_{i}=-\overline{\bar{Y}}_{i} \frac{d}{d y} \tilde{\overrightarrow{E_{i}}}(n, y)
$$

where

$$
\overline{\bar{Y}}_{i}=\left(1 / \gamma_{y i}^{2}\right)\left[\begin{array}{cc}
-\alpha_{n} \beta & \gamma_{y i}^{2}-\beta^{2} \\
\alpha_{n}^{2}-\gamma_{y i}^{2} & \alpha_{n} \beta
\end{array}\right]
$$

From (11) the magnetic field is known as a function of the tangential electric field at the interface. Applying the corresponding boundary conditions,

$$
\left|\tilde{\vec{H}}_{i+1}(n, y)-\tilde{\vec{H}}_{i}(n, y)\right|_{y=h_{i}}=\overline{\bar{T}} \cdot \tilde{\overrightarrow{J_{i}}}(n) \quad \overline{\bar{T}}=\left(\begin{array}{rr}
0 & -1 \\
1 & 0
\end{array}\right)
$$

we obtain a linear relation between $\tilde{\vec{E}}_{i}(n)$ and $\tilde{\overrightarrow{J_{i}}}(n)$ and thus an expression for $\overline{\bar{g}}_{i, i}$ (for a biaxial anisotropic dielectric medium):

$$
\begin{aligned}
\overline{\bar{g}}_{i, i}(n)=\overline{\bar{T}}^{-1}\left\{\overline{\bar{Y}}_{i+1} \cdot \overline{\bar{K}}_{i+1} \cdot \operatorname{coth}\left(\overline{\bar{K}}_{i+1} H_{i+1}\right)\right. \\
\left.+\overline{\bar{Y}}_{i} \cdot \overline{\bar{K}}_{i} \cdot \operatorname{coth}\left(\overline{\bar{K}}_{i} H_{i}\right)\right\}
\end{aligned}
$$

\section{B. Evaluation of $\overline{\bar{g}}_{i, i+1}$}

In this case, the structure to be considered is shown in Fig. 2(c). The tangential electric field at the $(i+1)$ th interface and the corresponding surface current on the ground plane are related by

$$
j \omega \mu_{0} \tilde{\overrightarrow{J_{i}}}(n)=\overline{\bar{g}}_{i, i+1}(n) \cdot \tilde{\vec{E}}_{i+1} .
$$

Once again we apply the boundary conditions for $\vec{H}$ and find the expression for $\overline{\bar{g}}_{i, i+1}$ :

$$
\overline{\bar{g}}_{i, i+1}(n)=-\overline{\bar{T}}^{-1} \cdot \overline{\bar{y}}_{i+1} \cdot \overline{\bar{k}}_{i+1} \cdot\left[\sinh \left(\overline{\bar{k}}_{i+1} H_{i+j}\right)\right]^{-1} \text {. }
$$

From the reciprocity theorem,

$$
\overline{\bar{g}}_{i+j, i}(n)=\overline{\bar{g}}_{i, i+j}(n) \text {. }
$$

Now the linear relation in the spectral domain (9) is known. In the following section this expression will be used to derive the dyadic Green's function associated with the multilayer structure in Fig. 1. This function in conjunction with the Ritz-Galerkin method will provide the dispersion relation we are interested in. Note that (14) and (16) are even functions of $\overline{\bar{K}}$ and so they can be properly evaluated from a knowledge of the eigenvalues of $\overline{\bar{B}}$.

\section{The Spectral Dyadic Green's Function}

Assuming the presence of conductors at the $M$ th interface, the Fourier transforms of the surface current density and the tangential components of the electric field are related via the spectral dyadic Green's function:

$$
\begin{aligned}
j \omega \mu_{0} \tilde{\vec{J}}_{M}(n) & =\overline{\bar{L}}_{M}(n) \cdot \tilde{\vec{E}}_{M}(n) \\
\tilde{\vec{E}}_{M}(n) & =j \omega \mu_{0} \overline{\bar{G}}_{M}(n) \cdot \tilde{\overrightarrow{\vec{J}}}_{M}(n)
\end{aligned}
$$

with $\overline{\bar{L}}_{M}(n)^{-1}=\overline{\bar{G}}_{M}(n)=$ dyadic Green's function.
As mentioned in the previous section, expression (9) is of interest mainly because it provides a method for deriving a general form of the dyadic Green's function for multilayered configurations. Relation (9) is formally identical to the one reported in [27], so we can use a similar recurrence expression to evaluate the dyadic Green's function. Caution must be exercised in recognizing that the expressions for $\overline{\bar{g}}$ in [27] are scalar and in this paper are $2 \times 2$ dyadics. In this way, once $\overline{\bar{g}}$ matrices have been calculated for each layer and account is taken of the absence of currents at the interfaces without conductors, the inverse of the spectral dyadic Green's function can be obtained from the following recurrence formulas:

$$
\overline{\bar{L}}_{M}(n)=\overline{\bar{L}}_{M}^{B}(n)+\overline{\bar{L}}_{N-M}^{U}(n)-\overline{\bar{g}}_{M, M}(n)
$$

where

$$
\begin{gathered}
\overline{\bar{L}}_{i}^{B}=\overline{\bar{g}}_{i, i}-\overline{\bar{g}}_{i, i-1} \cdot\left(\overline{\bar{L}}_{i-1}^{B}\right)^{-1} \cdot \overline{\bar{g}}_{i-1, i} \\
\overline{\bar{L}}_{i}^{U}=\overline{\bar{g}}_{N-i, N-i}-\overline{\bar{g}}_{N-i, N-i+1} \cdot\left(\overline{\bar{L}}_{i-1}^{U}\right)^{-1} \cdot \overline{\bar{g}}_{N-i+1, N-i} \\
i>1 .
\end{gathered}
$$

The first term $(i=1)$ in the above relations depends on the nature of the upper and lower interfaces:

a) ground planes.

$$
\overline{\bar{L}}_{1}^{B}=\overline{\bar{g}}_{1,1} \quad \overline{\bar{L}}_{1}^{U}=\overline{\bar{g}}_{N-1, N-1}
$$

b) magnetic walls:

$$
\begin{gathered}
\overline{\bar{L}}_{1}^{B}=\overline{\bar{g}}_{1,1}-2 \overline{\bar{g}}_{1,0} \cdot\left[\overline{\bar{g}}_{0,0}\right]^{-1} \cdot \overline{\bar{g}}_{0,1} \\
\overline{\bar{L}}_{1}^{U}=\overline{\bar{g}}_{N-1, N-1}-2 \overline{\bar{g}}_{N-1, N} \cdot\left[\overline{\bar{g}}_{N, N}\right]^{-1} \cdot \overline{\bar{g}}_{N, N-1}
\end{gathered}
$$

with

$$
\begin{aligned}
\overline{\bar{g}}_{0,0} & =2 \overline{\bar{T}} \cdot \overline{\bar{Y}}_{1} \cdot \overline{\bar{K}}_{1} \cdot \operatorname{coth}\left[\overline{\bar{K}}_{1} H_{1}\right] \\
\overline{\bar{g}}_{N, N} & =2 \overline{\bar{T}} \cdot \overline{\bar{Y}}_{N} \cdot \overline{\bar{K}}_{N} \cdot \operatorname{coth}\left[\overline{\bar{K}}_{N} H_{N}\right] .
\end{aligned}
$$

These expressions lead to a computer subroutine whose input variables are the tensor permittivities and thicknesses of the dielectric sheets. The spectral Green's function is then obtained for any layered geometry using this subroutine. Because of the form of the described derivation process, the asymptotic behavior of the Green's function is easily obtained. This behavior coincides with the one of the structure shown in Fig. 2(b). That is, although the Green's function for a general multilayer structure is very complex, its asymptotic part reduces to the Green's function of a simpler equivalent structure [18]. This fact is exploited in the numerical implementation of the method.

\section{Numerical Results}

The dispersion equation for the generalized structure considered in this paper is obtained by applying the conventional Ritz-Galerkin procedure. The variational expressions used as starting points involve either the surface current density on the strips (striplike configurations) or the tangential electric fields in the slots (slotlike and finline 


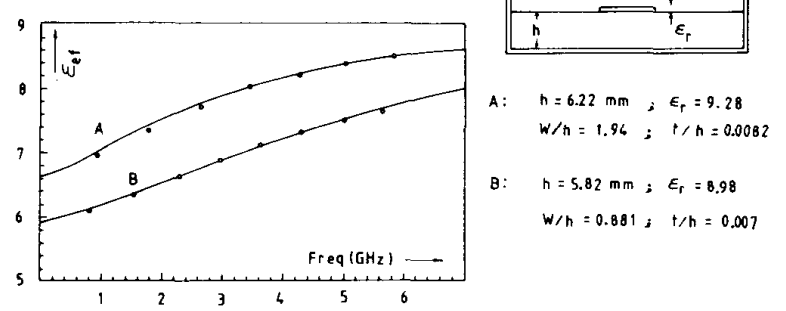

Fig. 3. Comparison between our results ( - ) and the measurements reported in [30] (०) for the large-scale microstrip line shown in the figure.

configurations). In the spectral domain these expressions are as follows.

Striplike systems:

$$
\sum_{n=-\infty}^{+\infty} \tilde{\overrightarrow{\vec{J}_{M}^{\prime}}}(n) \cdot \overline{\bar{G}}_{M}(n) \cdot \tilde{\overrightarrow{\vec{J}_{M}^{*}}}(n)=0 .
$$

Slotlike or finline systems:

$$
\sum_{n=-\infty}^{+\infty} \tilde{\vec{E}}_{M}^{t}(n) \cdot \overline{\bar{L}}_{M}(n) \cdot \tilde{\vec{E}}_{M}^{*}(n)=0 .
$$

As is well known, a proper choice of the basis functions approximating the actual current or field distributions is critical at this point of the analysis. Following the suggestions of Jansen [28], the experience furnished by other authors, and our own numerical experiments, the following set of basis functions has been found especially suitable for computational purposes:

$$
\begin{aligned}
& f_{m}^{i}(x)=\frac{T_{m}\left(\frac{x-s_{i}}{w_{i} / 2}\right)}{\sqrt{1-\left(\frac{x-s_{i}}{w_{i} / 2}\right)^{2}}} \\
& g_{m}^{i}(x)=j U_{m}\left(\frac{x-s_{i}}{w_{i} / 2}\right) \sqrt{1-\left(\frac{x-s_{i}}{w_{i} / 2}\right)^{2}}
\end{aligned}
$$

with

$$
\begin{aligned}
& J_{M z}(x), E_{M x}(x)=\sum_{i} \sum_{m} a_{m}^{i} f_{m}^{i}(x) \\
& J_{M x}(x), E_{M z}(x)=\sum_{i} \sum_{m} b_{m}^{i} g_{m}^{i}(x)
\end{aligned}
$$

where $s_{i}$ is the central coordinate of the $i$ th strip (slot), $w_{i}$ is the strip (slot) width, and $T_{m}(x), U_{m}(x)$ are Chebyshev polynomials of the first and second kind. (Note that the edge condition is explicitly taken into account.)

The application of the Ritz-Galerkin method leads to a homogeneous system of linear equations for the expansion coefficients in (25) which has a nontrivial solution when the determinant of the coefficient matrix vanishes. This

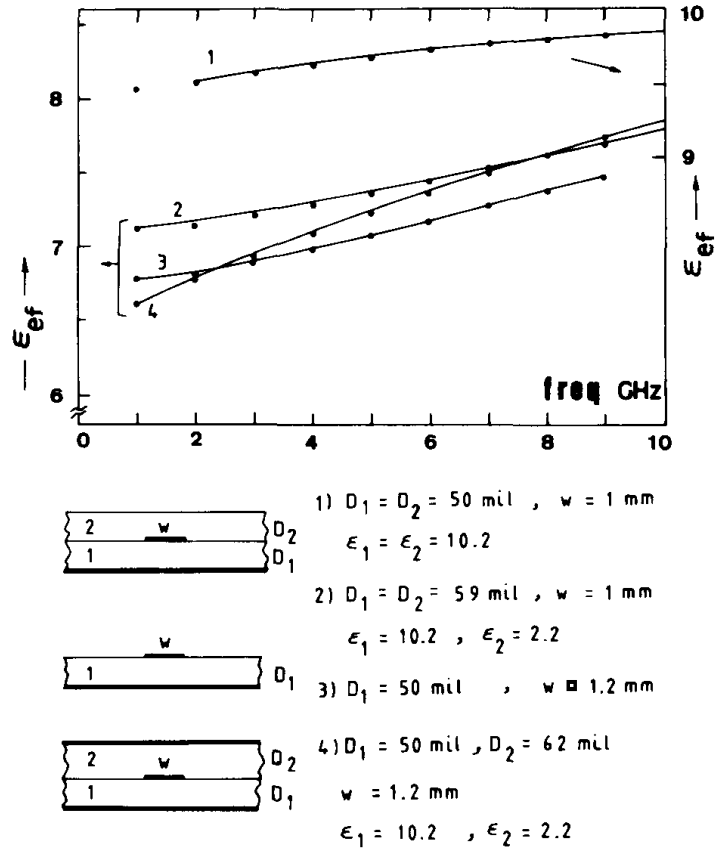

Fig. 4. Effective dielectric constants for several microstrip configurations as a function of frequency. Solid lines are with the results in [18]; circle points have been computed with our programs.

condition provides the dispersion relation in numerical form.

The singular behavior of the functions in (25) at the end of the conductors spreads out their Fourier spectrum in such a way that the series defining the entries of the Ritz-Galerkin matrix are very slowly convergent. In order to gain numerical efficiency it is necessary to overcome this difficulty. Fortunately, with this choice of basis functions, the asymptotic behavior of the above-mentioned series can be extracted and added analytically or reduced to an extremely fast converging series. The difference between the original series and its asymptotic part is added numerically, but its convergence is much better than that of the original one. It is important to emphasize that all series appearing in the computations present the same asymptotic behavior in such a way that these computations must be performed only once. In this way, the most time consuming part of the SDA is substantially improved. This mathematical preprocessing requires a knowledge of the asymptotic behavior of the spectral Green's function, which is very complex if several dielectric layers are present. Nevertheless, as discussed above, it must be noted (in accordance with [18]) that this behavior depends only on the dielectric layers immediately adjacent to the interface with conductors. The contribution of the remaining layers decreases exponentially with $\alpha_{n}$, as can be seen from the form of (16).

Using the theory in this paper and taking into account the above considerations, we have written two computer programs to calculate the propagation constants of both generalized microstrip and generalized finline or slotlike 


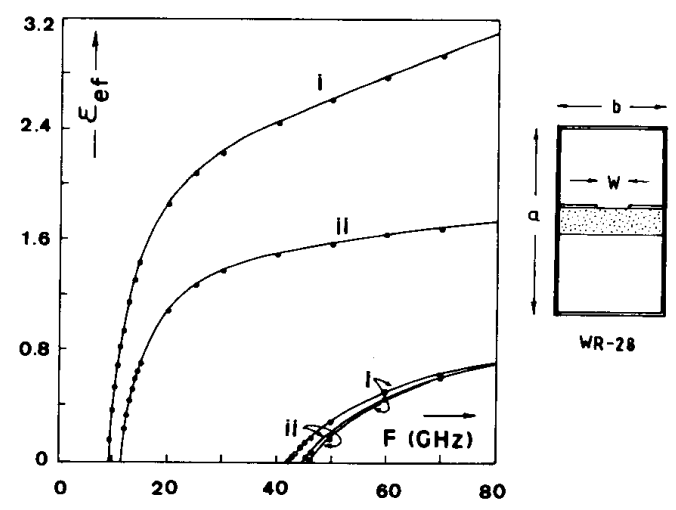

(a)

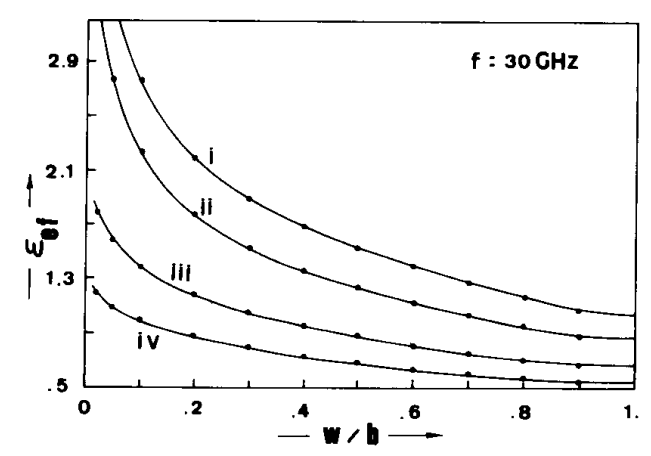

(b)

Fig. 5. In this figure we compare our results (circle points) with the ones recently reported by Shalaby et al. [20] (solid lines) for the unilateral finline on uniaxial anisotropic substrate drawn in the figure. (a) Dispersion characteristics of the first three odd modes for a finline on (i) sapphire and (ii) boron nitride substrates. $W / b=0.1$; substrate thickness $=0.125 \mathrm{~mm}$. (b) Slot width dependence of the effective dielectric constant for the dominant mode for a finline on four different substrates: (i) Epsilam-10, (ii) sapphire, (iii) boron nitride, (iv) $\epsilon_{r}=2.2$. The substrate thickness and the waveguide are the same as in (a).

structures. In order to check the computer programs, we have made exhaustive comparisons with previously published results. First, we have checked the low-frequency values with those obtained with the method reported in [3], obtaining agreement better than 0.5 percent. We then compared our results with other results obtained using very accurate methods. For instance, the results have been checked against those given by Kretch and Collin [29] (microstrip line on isotropic and anisotropic substrates), and they agree to within the accuracy with which data can be read from the graphs. The results obtained with our programs are also indistinguishable from the ones reported in [11, fig. 2] and [26, table I]. We have also made comparisons with experimental data reported in the literature. For instance, we have found excellent agreement between our results and the measurements reported by Deibele et al. [30] for a large-scale microstrip model on an isotropic substrate (Fig. 3). This fact confirms the accuracy of the programs for very high frequencies. Fig. 4 shows a comparison with the results recently reported by Das and Pozar

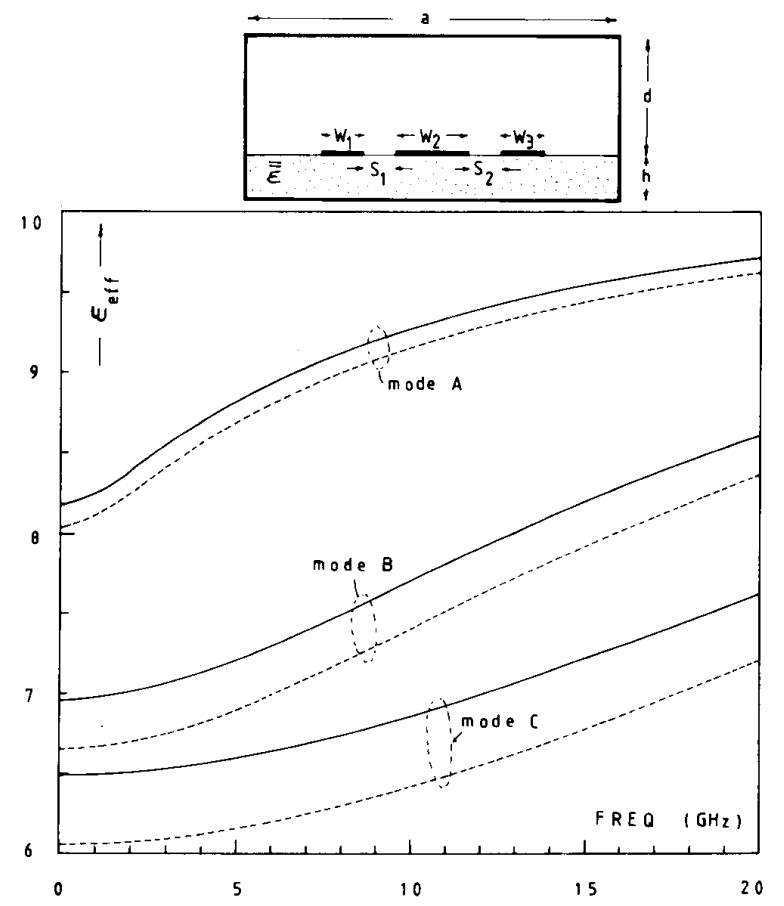

Fig. 6. Dispersive behavior of the three quasi-TEM modes of the three coupled strips on Epsilam-10 substrate shown in the figure. Solid lines have been computed taking into account the anisotropy of the substrate $\left(\epsilon_{x}=13.0, \epsilon_{y}=10.3\right)$ while in dashed lines the substrate has been assumed to be isotropic, with $\epsilon_{r}=10.3$. Discrepancies are not negligible at all. $a=20 \mathrm{~mm} ; d=10 \mathrm{~mm} ; h=1 \mathrm{~mm} ; W_{1}=W_{3}=1 \mathrm{~mm} ; W_{2}=2$ $\mathrm{mm} ; S_{1}=S_{2}=0.5 \mathrm{~mm}$.

[18] for several multilayer microstrip structures, and in Fig. 5 the comparison refers to a unilateral finline recently studied by Shalaby et al. [20] for different iso/anisotropic substrates. Very good agreement with previously published results [6] has also been found for higher order modes.

Although only a few results have been included, we have also made comparisons with many other numerical and graphical data reported in the works cited in this paper and certain others not cited here. In most cases the agreement was very satisfactory. From this, we can use the developed programs with confidence. As an example, in Fig. 6 we show the mode effective dielectric constants for a three-strip configuration on Epsilam-10. The data have been calculated both neglecting the anisotropy of the substrate and taking it into account. As can be seen from the graphs, the effect of the anisotropy is not negligible. This effect is very important in many structures used in practice and so the electric anisotropy of the materials used as substrates should be considered in the computation of the propagation characteristics of the lines. Another example can be viewed in Fig. 7. Even- and odd-mode effective dielectric constants for two symmetrically coupled strips on P.B.N. are shown. The difference between the mode phase velocities is significantly reduced using a thin overlay made of the same material, as can be seen from the figure. This result could be useful in coupler design. 


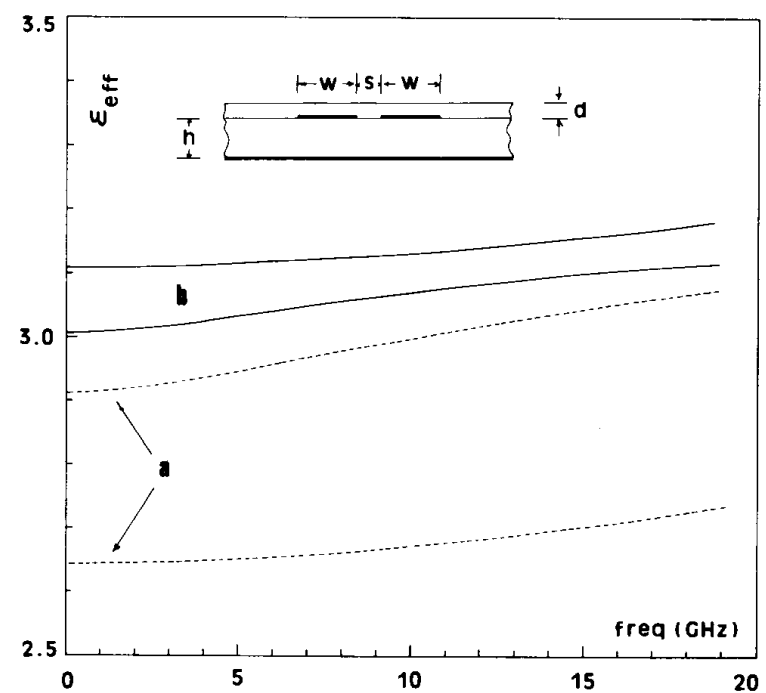

Fig. 7. Even- and odd-mode effective dielectric constant for (a) coupled strips on P.B.N. $(d=0)$ and (b) the same structure covered with a P.B.N. overlay $(d=0.08 \mathrm{~mm}) . \epsilon_{x}=\epsilon_{z}=5.12 ; \epsilon_{y}=3.40 ; w=1.2 \mathrm{~mm}$; $s=0.5 \mathrm{~mm} ; h=0.635 \mathrm{~mm}$

\section{CONCLUSIONS}

A unified theoretical and numerical analysis of generalized planar or quasi-planar structures is presented in this paper. This analysis allows us to compute the propagation constants of fundamental and higher order modes for these types of structures taking into account uniaxial and biaxial anisotropic dielectrics and an arbitrary number of dielectric layers. The analysis is achieved using the spectraldomain approach, which is found to yield excellent accuracy if a judicious choice of trial functions is made. The spectral Green's function is computed, using a simple recurrence expression, from the solutions of two elementary one- and two-layer problems. Each dielectric layer is characterized by its transverse propagation matrix in such a way that a new dielectric layer means only the introduction of a simple $2 \times 2$ matrix in the analysis. The dispersion solution is obtained by applying the Ritz-Galerkin method, using as unknowns the surface current densities or the tangential electric fields depending on the type of structure to be analyzed (microstrip or finline, respectively). The asymptotic behavior of the series defining the entries of the Ritz-Galerkin matrix must be explicitly incorporated in order to accelerate the convergence and gain accuracy. The results obtained with the theory in this paper compare very well with previously published data for particular structures.

\section{REFERENCES}

1] N. G. Alexopoulos, "Integrated circuit structures on anisotropic substrates," IEEE Trans. Microwave Theory Tech., vol. MTT-33, pp. 847-881, Oct. 1985.

[2] F. Medina, "Study of generalised shielded planar transmission lines on anisotropic dielectrics" (in Spanish), Ph.D. thesis, Electronics and Electromagnetics Department, Univ. of Seville, Spain, 1987.

[3] F. Medina and M. Horno, "Capacitance and inductance matrices for multistrip structures in multilayered anisotropic dielectrics,"
IEEE Trans. Microwave Theory Tech., vol. MTT-35, pp. 1002-1008, Nov. 1987.

[4] D. Gelder, "Numerical determination of microstrip properties using the transverse field components," Proc. Inst. Elec. Eng., vol. 117 , no. 4 , pp. $699-703$, Apr. 1970

[5] H. Baudrand, M. Boussouis, and J. L. Amalric, "Analysis of some planar structures by the least squares boundary residual method," IEEE Trans. Microwave Theory Tech., vol. MTT-34, pp. 298-301, Feb. 1986.

[6] E. Yamashita and K. Atsuki, "Analysis of microstrip-like transmission lines by nonuniform discretization of integral equations," IEEE Trans. Microwave Theory Tech., vol. MTT-24, pp. 195-200, Apr. 1976.

[7] B. M. Sherrill and N. G. Alexopoulos, "The method of lines applied to a finline/strip configuration on an anisotropic substrate," IEEE Trans. Microwave Theory Tech., vol. MTT-35, pp. 568-575, June 1987.

[8] J. Bornemann, "Rigourous field theory analysis of quasiplanar waveguides," Proc. Inst. Elec. Eng., vol. 132, pt. H, no. 1, pp. 1-6, Feb. 1985.

[9] A. K. Saad and K. Schunemann, "Efficient eigenmode analysis for planar transmission lines," IEEE Trans. Microwave Theory Tech., vol. MTT-30, pp. 2125-2131, Dec. 1981.

[10] G. E. Mariki and C. Yeh, "Dynamic three-dimensional TLM analysis of microstriplines on anisotropic substrate," IEEE Trans. Microwave Theory Tech., vol. MTT-33, pp. 789-799, Sept. 1985.

[11] A.-M. A. El-Sherbiny, "Hybrid mode analysis of microstrip lines on anisotropic substrates," IEEE Trans. Microwave Theory Tech., vol. MTT-29, pp. 1261-1266, Dec. 1981.

[12] K. Kawano, "Hybrid mode analysis of a broadside-coupled microstripline," Proc. Inst. Elec. Eng., pt. H, vol. 131, no. 1, pp. 21-24, Feb. 1984

[13] B. J. Janiczak, "Phase constant characteristics of generalised asymmetric three-coupled microstrip lines," Proc. Inst. Elect. Eng., pt. H, vol. 132, no. 1, pp. 23-26, Feb. 1985.

[14] L.-P. Schmidt and T. Itoh, "Characteristics of unilateral finline structures with arbitrarily located slots," IEEE Trans. Microwave Theory Tech., vol. MTT-29, pp. 352-355, Apr. 1981.

[15] H. C. C. Fernandes and A. J. Giarola, "Dispersion in unilateral finlines with two dielectric layers," Proc. Inst. Elect. Eng., pt. H, vol. 131, no. 3, pp. 139-142, June 1984

[16] T. Itoh, "Spectral domain immitance approach for dispersion characteristics of generalized printed transmission lines," IEEE Trans. Microwave Theory Tech., vol. MTT-28, pp. 733-736, July 1980.

[17] J. B. Davies and D. Mirshekar-Syahkal, "Spectral domain solution or arbitrary coplanar transmission line with multilayer substrate," IEEE Trans. Microwave Theary Tech., vol. MTT-25, pp. 143-146, Feb. 1977.

[18] N. K. Das and D. M. Pozar, "A generalized spectral-domain Green's function for multilayer dielectric substrates with application to multilayer transmission lines," IEEE Trans. Microwave Theory Tech., vol. MTT-35, pp. 326-335, Mar. 1987.

[19] T. Kitazawa and Y. Hayashi, "Propagation characteristics of striplines with multilayered anisotropic media," IEEE Trans. Microwave Theory Tech., vol. MTT-31, pp. 429-433, June 1983.

[20] A.-A. T. K. Shalaby and A. Kumar, "Dispersion in unilateral fin-lines on anisotropic substrates," IEEE Trans. Microwave Theory Tech., vol. MTT-35, pp. 448-450, Apr. 1987.

[21] M. R. G. Maia, A. G. D’Assunçao, and A. J. Giarola, "Dynamic analysis of microstrip lines and finlines on uniaxial anisotropic substrates," IEEE Trans. Microwave Theory Tech., vol. MTT-35, pp. 881-886, Oct. 1987 .

[22] H. Lee and V. K. Tripathi, "Spectral domain analysis of frequency dependent propagation characteristics of planar structures on uniaxial medium," IEEE Trans. Microwave Theory Tech., vol. MTT-30, pp. 1188-1193, Aug. 1982.

[23] C. M. Krowne, "Fourier transformed matrix method of finding propagation characteristics of complex anisotropic layered media," IEEE Trans. Microwave Theory Tech., vol. MTT-32, pp. 1617-1625, Dec. 1984.

[24] R. Marqués and M. Horno, "Dyadic Green's function for microstrip-like transmission lines on a large class of anisotropic substrates," Proc. Inst. Elec. Eng., pt. H, vol. 133, pp. 450-454, Dec. 1986.

[25] R. Marqués and M. Horno, "On the spectral dyadic Green's function for stratified linear media. Application to multilayer MIC 
lines with anisotropic dielectrics," Proc. Inst. Elec. Eng., pt. H, vol. 134, pp. 241-247, June 1987.

[26] H.-Y. Yang and N. G. Alexópoulos, "Uniaxial and biaxial substrate effects on finline characteristics," IEEE Trans. Microwave Theory Tech., vol. MTT-35, pp. 24-29, Jan. 1987.

[27] F. Medina and M. Horno, "Upper and lower bounds on mode capacitances for a large class of anisotropic multilayered microstrip-like transmission lines," Proc. Inst. Elec. Eng., pt. H, vol. 132, pp. 157-163, June 1985

[28] R. H. Jansen, "The spectral domain approach for microwave integrated circuits," IEEE Trans. Microwave Theory Tech., vol MTT-33, pp. 1043-1056, Oct. 1985.

[29] B. E. Kretch and R. E. Collin, "Microstrip dispersion including anisotropic substrates," IEEE Trans. Microwave Theory Tech., vol. MTT-35, pp. 710-718, Aug. 1987.

[30] S. Deibele and J. B. Beyer, "Measurements of microstrip effective relative permittivities," IEEE Trans. Microwave Theory Tech., vol. MTT-35, pp. 535-538, May 1987.

区

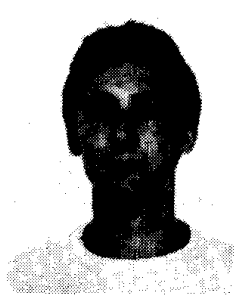

Francisco Medina was born in Puerto Real, Cádiz, Spain, on November 9, 1960. He received the Licenciatura degree in physics in September 1983 from the University of Sevilla, Sevilla, Spain. In September 1987 he received a doctorate in physics from the same University.

He is currently Assistant Lecturer of Electric- ity and Magnetism in the Department of Electronics and Electromagnetism, University of Sevilla. His research interests focus on multiconductor planar transmission lines and MIC design.

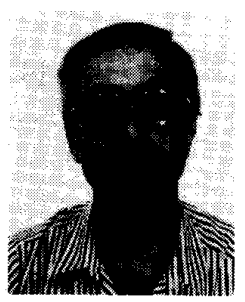

Manuel Horno (M'75) was born in Torre del Campo, Jaén, Spain. He received the degree of Licenciado in Physics in June 1969 and the degree of Doctor en Ciencias in physics in January 1972, both from the University of Sevilla, Spain.

Since October 1969 he has been with the Department of Electricity and Electronics at the University of Sevilla, where he became an Assistant Professor in 1970, an Associate Professor in 1975, and Professor in 1986. His main fields of interest include boundary value problems in electromagnetic theory, wave propagation through anisotropic media, and microwave integrated circuits. He is presently engaged in the analysis of planar transmission lines embedded in anisotropic materials, multiconductor transmission lines, and planar slow-wave structures.

急

Henri Baudrand (M'86) was born in 1939. He obtained the "Diplôme d'Ingénieur" in electronics and the Docteur-es-Sciences degree in $\mathrm{mi}$ crowaves, both from E.N.S.E.E.I.H.T., Toulouse, France, in 1962 and 1966 , respectively.

Since then, he has been working on active and passive microwave integrated circuits in the Laboratoire de Microondes of E.N.S.E.E.I.H.T., where he is a Professor of Physics. 